\title{
A mononucleotide markers panel to identify hMLH1/hMSH2 germline mutations
}

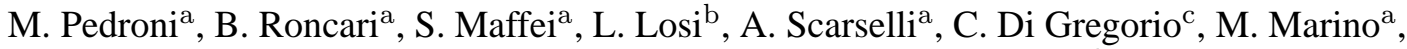 \\ L. Roncucci ${ }^{\mathrm{a}}$, P. Benatti ${ }^{\mathrm{a}}$, G. Ponti ${ }^{\mathrm{a}}$, G. Rossi ${ }^{\mathrm{a}}$, M. Menigatti ${ }^{\mathrm{a}}$, A. Viel $^{\mathrm{d}}$, M. Genuardi $^{\mathrm{e}}$ and \\ M. Ponz de Leon ${ }^{\mathrm{a}, *}$ \\ ${ }^{a}$ Department of Medicine and Medical Specialities, University of Modena and Reggio Emilia, Modena, Italy \\ ${ }^{\mathrm{b}}$ Department of Pathology, University of Modena and Reggio Emilia, Modena, Italy \\ ${ }^{\mathrm{c}}$ Division of Anatomical Pathology, Carpi Hospital, Modena, Italy \\ ${ }^{\mathrm{d}}$ Division of Experimental Oncology I, IRCCS, Aviano, Italy \\ ${ }^{\mathrm{e}}$ Department of Clinical Fysiopathology, Medical Genetics Section, University of Firenze, Italy
}

\begin{abstract}
Hereditary NonPolyposis Colorectal Cancer (Lynch syndrome) is an autosomal dominant disease caused by germline mutations in a class of genes deputed to maintain genomic integrity during cell replication, mutations result in a generalized genomic instability, particularly evident at microsatellite loci (Microsatellite Instability, MSI). MSI is present in $85-90 \%$ of colorectal cancers that occur in Lynch Syndrome. To standardize the molecular diagnosis of MSI, a panel of 5 microsatellite markers was proposed (known as the "Bethesda panel"). Aim of our study is to evaluate if MSI testing with two mononucleotide markers, such as BAT25 and BAT26, was sufficient to identify patients with $h M L H 1 / h M S H 2$ germline mutations. We tested 105 tumours for MSI using both the Bethesda markers and the two mononucleotide markers BAT25 and BAT26. Moreover, immunohistochemical evaluation of MLH1 and MSH2 proteins was executed on the tumours with at least one unstable microsatellite, whereas germline $h M L H 1 / h M S H 2$ mutations were searched for all cases showing two or more unstable microsatellites. The Bethesda panel detected more MSI $(+)$ tumors than the mononucleotide panel ( $49.5 \%$ and $28.6 \%$, respectively). However, the mononucleotide panel was more efficient to detect MSI $(+)$ tumours with lack of expression of Mismatch Repair proteins (93\% vs 54\%). Germline mutations were detected in almost all patients whose tumours showed MSI and no expression of MLH1/MSH2 proteins. No germline mutations were found in patients with MSI $(+)$ tumour defined only through dinucleotide markers. In conclusion, the proposed mononucleotide markers panel seems to have a higher predictive value to identify $h M L H 1$ and $h M S H 2$ mutation-positive patients with Lynch syndrome. Moreover, this panel showed increased specificity, thus improving the cost/effectiveness ratio of the biomolecular analyses.
\end{abstract}

Keywords: MSI, HNPCC, colorectal cancer, MLH1, MSH2

\section{Introduction}

Hereditary Non-Polyposis Colorectal Cancer (HN$\mathrm{PCC})$ is a common autosomal dominant disease associated with an increased lifetime risk for cancer, especially colorectal and endometrial carcinomas [1] HN-

\footnotetext{
*Corresponding author: Maurizio Ponz de Leon, MD, Dipartimento di Medicine e Specialità Mediche, Medicina I, Policlinico, Via del Pozzo 71, 41100 Modena, Italy. Tel.: +39 59 4222269; Fax: +3959 4222958; E-mail: deleon@unimo.it.
}

PCC accounts for approximately $2-5 \%$ of all colorectal tumours and is characterized by an early age of cancer onset and by the frequent development of multiple tumours. This familial cancer syndrome is caused by germline mutations in genes encoding components of the post-replication DNA Mismatch Repair system (DNA MMR system) [2]. DNA MMR system is required for correction of mismatches that occur during replication, and is composed by at least five genes: hMLH1, hMSH2, hMSH6, hMSH3, hPMS2 [3,4]. In the majority of families with HNPCC, germline muta- 
tions affect $h M L H 1$ or $h M S H 2$ [5], though mutation have also been reported in $h M S H 6$ and $h P M S 2$ gene [6]. Inactivation of MMR genes results in a high frequency of small deletions or insertions with simple repeat sequences that occur ubiquitously throughout the genome (microsatellites) and is referred to as Microsatellite Instability (MSI).

A total 85-90\% of HNPCC patients show MSI $(+)$ tumours, whereas MSI is present in only $10-15 \%$ of sporadic colorectal tumours [7]. Since HNPCC-associated colorectal malignancies are characterized by MSI, to test a tumour for microsatellite instability is an important tool to identify these families. To standardize the molecular definition of MSI, in December 1997 a National Cancer Institute Workshop on HNPCC recommended a panel of five microsatellite markers; the panel included two mononucleotide repeats (BAT25 and BAT26) and three dinucleotide repeats (D2S123, D5S346 and D17S250). Using this panel, usually referred to as the "Bethesda panel" high-frequency Microsatellite Instability (MSI-H) was defined as having instability in two or more markers, whereas lowfrequency (MSI-L) and microsatellite stable (MSS) were defined as having instability in one or zero markers, respectively. The 2002 Consensus meeting recognized that dinucleotide markers were less sensitive than mononucleotide to identify MSI-H tumours and that mononucleotide repeats should replace dinucleotide in the panel [8]. The use of mononucleotide repeats such as BAT25 (an intronic $\mathrm{T}_{25}$ sequence in the $c$-KIT gene) and BAT26 (an intronic $\mathrm{A}_{26}$ sequence in the $h M S H 2$ gene) has proven to be very useful for the identification of the MSI-H group of tumours [9]. Instability at these loci appears in the majority of neoplasms defined as MSI-H, but rarely in MSI-L tumours [10]. Therefore, the exclusive use of mononucleotide repeats seems sufficient to detect true MSI [11]. It is noteworthy that $h M L H 1$ and $h M S H 2$ germline mutations give rise to "typical" HNPCC families that fulfil the so called "Amsterdam criteria", and show a high degree of MSI in the tumours [12]. In addition, recent studies have shown that MSI can be an important prognostic marker, since MSI-H colorectal cancers are often associated with a more favourable prognosis after surgical resection [13, 14]. Moreover, there is evidence suggesting that colorectal cancer patients with MSI-H tumours might respond differently to 5FU-based chemotherapy. Thus the assessment of the MSI status in colorectal cancer can be of relevance for at least three purposes: first, as a screening tool for HNPCC; second, as a prognostic marker; and finally, as a potential predictive factor of response to chemotherapy.
The accuracy of MSI as a marker to identify colon cancer that has lost MMR function is depending on the technical details of the assay and, in particular, on the type of markers used to detect MSI. The aim of the study was to evaluate the specificity and sensitivity of a mononucleotide marker panel, composed by BAT25 and BAT26, in order to identify $h M L H 1$ and $h M S H 2$ mutation-positive HNPCC patients. For these purposes, we compared the proposed panel with the classical "Bethesda panel" in a series of 105 colorectal neoplasms from HNPCC or "Suspected HNPCC" families.

\section{Patients and methods}

\subsection{Patients}

The patients described in this study were recruited through the specialized colorectal cancer Registry of the Health Care District 16, instituted in 1984, which included the town of Modena (Northern Italy) and 10 surrounding communities. We identified HNPCC patients following a previously described multistep approach [15]. Briefly, the nuclear pedigrees of all incident cases of colorectal cancer diagnosed during the period 1984-2003 were collected and stratified according to the presence of some clinical features, all indicative of an increased risk of HNPCC (such as "verticality", aggregation of cancer in a given sibship, early age of cancer onset, presence of multiple primaries, localization in the right colon and mucinous histological type). When a patient had 2 or more of these features, an extended genealogic tree was traced and then analyzed for the presence of diagnostic criteria for HNPCC (Amsterdam criteria II) [16]. All together we could identify 36 HNPCC kindreds and 75 families which fulfilled the clinical criteria of suspected HNPCC suggested by Park and coworkers [17]. According to these authors, suspected HNPCC is defined as a kindred with a) at least two HNPCC-associated cancers in first-degree relatives (colorectum, endometrium, small intestine, urinary tract), b) multiple colorectal tumours, or at least one HNPCC-associated cancer diagnosed before the age of 50 years, or development of tumours of the Lynch spectrum among family members. A total of 105 eligible and unrelated probands with colorectal cancer were recruited in this study, 33 of whom were from HNPCC families whereas the remaining 72 were from suspected HNPCC. 


\subsection{Microdissection and DNA extraction}

Formalin-fixed, paraffin-embedded normal and tumour tissues were taken from all patients investigated. At least one tissue block representative of the tumour and one separate block containing normal colonic mucosa could be obtained. Serial sections from paraffinembedded matched normal and neoplastic primary tissue $(5 \mu \mathrm{m})$ were stained with Hematoxylin-eosin (H\&E); representative normal and tumour regions were identified by microscopic examination. Area of tumour tissue with more than $80 \%$ of malignant cells were selected in all cases. Ten of these selected areas were manually scraped off using a surgical scalpel under stereomicroscopic guidance and placed into microcentrifuge tubes. Xilene was added to each tube to dissolve paraffin. The resulting samples were then incubated overnight at $50^{\circ}$ in a Tween 20 -proteinase $\mathrm{K}$ lysis buffer. After digestion the DNA was purified with a NaCl-saturated solution and precipitated in absolute ethanol. Before using a template DNA for PCR analysis, heat inactivation of proteinase $\mathrm{K}$ was carried out at $80^{\circ}$ for 10 minutes.

\subsection{MSI analysis}

The MSI status of 105 tumours was evaluated using the full basic set of five markers (BAT25, BAT26, D2S123, D5S346, D17S250) and was defined according to the Bethesda guideline. It is noteworthy that the panel includes also the two mononucleotide markers BAT25 and BAT26. Using the mononucleotide markers panel, a tumour was defined as $\operatorname{MSI}(+)$ when showed instability with both markers and MSI(-) when showed no instability. DNA from normal and tumour tissue was amplified in a $10 \mu \mathrm{l}$ volume containing 30-50 ng of DNA, 0.15 pmol of dye-labelled forward and unlabeled reverse primers, $2 \mathrm{mM}$ concentration of each deoxynucleotide triphosphate, $1.5 \mathrm{mM} \mathrm{MgCl}_{2}, 50 \mathrm{mM}$ $\mathrm{KCl}, 10 \mathrm{mM}$ Tris (pH 8.3) and 0.6 units of Taq polymerase. Thermocycling conditions were: $94^{\circ} \mathrm{C}, 4 \mathrm{~min}$, followed by 11 touchdown cycles, each with a denaturing step at $94^{\circ} \mathrm{C}$ for $30 \mathrm{~s}$, an extension step at $72^{\circ} \mathrm{C}$ for $15 \mathrm{~s}$ and a $75 \mathrm{~s}$ annealing step that decreased $1^{\circ} \mathrm{C} /$ cycle (beginning at $65^{\circ} \mathrm{C}$ in the first cycle and decreasing to $55^{\circ} \mathrm{C}$ in the eleventh cycle). The eleventh cycle was then repeated 26 times for a total of 37 cycles of PCR; finally an extension step of $4 \mathrm{~min}$ at $72{ }^{\circ} \mathrm{C}$ followed by storage at $4^{\circ} \mathrm{C}$. PCR products were prepared for analysis by pooling $2 \mu \mathrm{l}$ of dye- $\mathrm{D}_{2}$ reaction, $1 \mu \mathrm{l}$ of dye$\mathrm{D}_{3}$ and $0.5 \mu \mathrm{l}$ of dye- $\mathrm{D}_{4}$ reaction; $40 \mu \mathrm{l}$ of deionized formamide and $0.5 \mu$ l of CEQ DNA size standard-400 were added to $0.7 \mu \mathrm{l}$ of the each mixture. All samples were run on CEQ 8000 sequencer and analyzed using CEQ 8000 Fragment Analysis System by Beckman Coulter.

\subsection{Immunohistochemical analysis}

Immunohistochemical evaluation (IHC) of MSH2 and MLH1 protein expression was carried out on paraffin-embedded tissue sections of all tumours with at least one unstable microsatellite. MSI-L tumours were also analyzed for the expression of MSH6 protein. Slides with $5 \mu \mathrm{m}$ sections containing tumour tissue and normal colonic mucosa were deparaffinized in xilene and rehydrated in graded alcohols. Endogenous peroxidase was blocked by $3 \%$ hydrogen peroxide and antigen was revealed with $10 \mathrm{mM}$ citrate buffer $(\mathrm{pH}$ 6.0) at $350 \mathrm{~W}$ for $30 \mathrm{~min}$, by microwave. Mouse monoclonal antibodies to full-length MLH1 and MSH2 (clone G168-15, G129-1129, PharMingen, San Diego, CA) were used at a 1:100 dilution, while mouse monoclonal antibody against MSH6 protein (clone 44 Transduction Italia) at a 1:2000 dilution, overnight. Immunostaining was executed by the avidin-biotin peroxidase technique; diaminobenzidine was used as a chromogen. Slides were counterstained with hematoxylin. Normal tissue and stromal cells or lymphocytes adjacent to the respective tumour were used as internal positive controls. Loss of MLH1 and MSH2 protein expression was defined as complete absence of nuclear staining in tumour cells (but maintained in normal epithelial and stromal cells).

\subsection{Analysis of germline mutations in hMLH1 and hMSH2}

In all patients whose tumours were $\mathrm{MSI}(+)$ and with no MLH1/MSH2 protein expression, a search for germline mutations of the main MMR genes was carried out by direct genomic sequencing of all exons. Sequencing was executed on the basis of the immunohistochemical results. All patients with MSI-H tumours and no MLH1 protein expression in the tumours had previously been evaluated for a Northern Italian founder mutation of the $h M L H 1$ gene described of by our group [18]. Molecular analysis of both $h M L H 1$ and $h M S H 2$ genes was extended also to MSI $(+)$ cases that had normal expression of the main MMR proteins. Amplification products were generated with primers located in the flanking introns approximately 
Table 1

MSI analysis in the 105 colorectal tumours according to two different marker panels and expression of MLH1-MSH2 protein in MSI(+) tumours

\begin{tabular}{lcccrc}
\hline & MSI $(+)$ & MSI $(-)$ & no expression & expression & total MSI $(+)$ \\
\hline Bethesda Panel & $52(49.5 \%)$ & $39(37.14 \%)^{*}$ & $28(54 \%)$ & $24(46 \%) \S$ & 52 \\
Mononucleotide panel & $30(28.6 \%)$ & $75(71.4 \%)^{*}$ & $28(93 \%)$ & $2(75 \%) \S$ & 30 \\
\hline${ }^{*} \chi^{2}=16.35 ; p<0.001$. & & & & \\
$\S F_{1}=13.70 ; p<0.001$. & & & &
\end{tabular}

Table 2

MSI(+) tumours detected with the mononucleotide panel, expression of MMR protein and germline mutations

\begin{tabular}{|c|c|c|c|c|c|c|}
\hline \multirow[t]{2}{*}{ Tumour } & \multirow[t]{2}{*}{ Clinical features } & \multicolumn{2}{|c|}{$\begin{array}{c}\text { MSI }(+) \text { with } \\
\text { mononucleotide panel }\end{array}$} & \multirow[t]{2}{*}{$\begin{array}{l}\text { MLH1protein } \\
\text { expression }\end{array}$} & \multirow[t]{2}{*}{$\begin{array}{l}\text { MSH2 protein } \\
\text { expression }\end{array}$} & \multirow[t]{2}{*}{ Germline mutation } \\
\hline & & BAT25 & BAT26 & & & \\
\hline 7 & HNPCC & + & + & - & + & + \\
\hline 15 & HNPCC & + & + & - & + & + \\
\hline 18 & S-HNPCC & + & + & + & - & nv \\
\hline 19 & S-HNPCC & + & + & - & + & nv \\
\hline 20 & S-HNPCC & + & + & - & + & nv \\
\hline 21 & S-HNPCC & + & + & - & + & nv \\
\hline 25 & S-HNPCC & + & + & - & + & + \\
\hline 26 & HNPCC & + & + & + & + & + \\
\hline 29 & HNPCC & + & + & + & - & + \\
\hline 32 & Muir-Torre & + & + & + & - & + \\
\hline 38 & HNPCC & + & + & - & + & + \\
\hline 40 & HNPCC & + & + & + & - & + \\
\hline 41 & HNPCC & + & + & + & + & + \\
\hline 43 & Muir-Torre & + & + & + & - & + \\
\hline 48 & HNPCC & + & + & + & - & + \\
\hline 56 & HNPCC & + & + & - & + & nv \\
\hline 58 & HNPCC & + & + & - & + & + \\
\hline 68 & HNPCC & + & + & - & + & + \\
\hline 71 & HNPCC & + & + & - & + & + \\
\hline 73 & HNPCC & + & + & - & + & + \\
\hline 77 & HNPCC & + & + & - & + & + \\
\hline 79 & HNPCC & + & + & + & - & + \\
\hline 80 & S-HNPCC & + & + & + & - & + \\
\hline 81 & HNPCC & + & + & + & - & + \\
\hline 84 & S-HNPCC & + & + & + & - & + \\
\hline 88 & HNPCC & + & + & + & - & + \\
\hline 89 & HNPCC & + & + & - & + & + \\
\hline 93 & HNPCC & + & + & - & + & + \\
\hline 98 & HNPCC & + & + & - & + & + \\
\hline 103 & HNPCC & + & + & - & + & + \\
\hline
\end{tabular}

not valued $=$ nv, Suspected HNPCC $=$ S-HNPCC.

50 base pair from the respective intron/exon borders to detect all possible splice junction mutations. Direct sequencing of the PCR products was obtained using Dye Terminator Cycle Sequencing Kit (CEQ DTCS Kit, Beckman Coulter) and reactions were run on an CEQ 8000 capillary sequencer (Beckman Coulter) according to the manufacturer's instructions. To exclude the possibility of large genomic deletions of $h M L H 1$ and $h M S H 2$, all patients with MSI-H were analyzed by Multiplex Ligation-dependent Probe Amplification (MLPA). The MLPA test for $h M L H 1$ and $h M S H 2$ was obtained from MCR-Holland, Amsterdam, the Nether- lands. Pathogenic mutations were detected twice and confirmed in a second blood sample of the patient. The purpose of the study was clearly explained and an informed consent was obtained.

\subsection{Statistics}

Pearson's $\chi 2$ or Fisher's exact tests were applied to the results as appropriate. All values of $p \leqslant 0.05$ were considered significant. Sensitivity and specificity for MSI markers and immunohistochemistry were defined using the germline mutations results as the gold stan- 
Table 3

MSI(+) tumours detected with the Bethesda panel, expression of MLH1/MSH2 protein and germline mutations

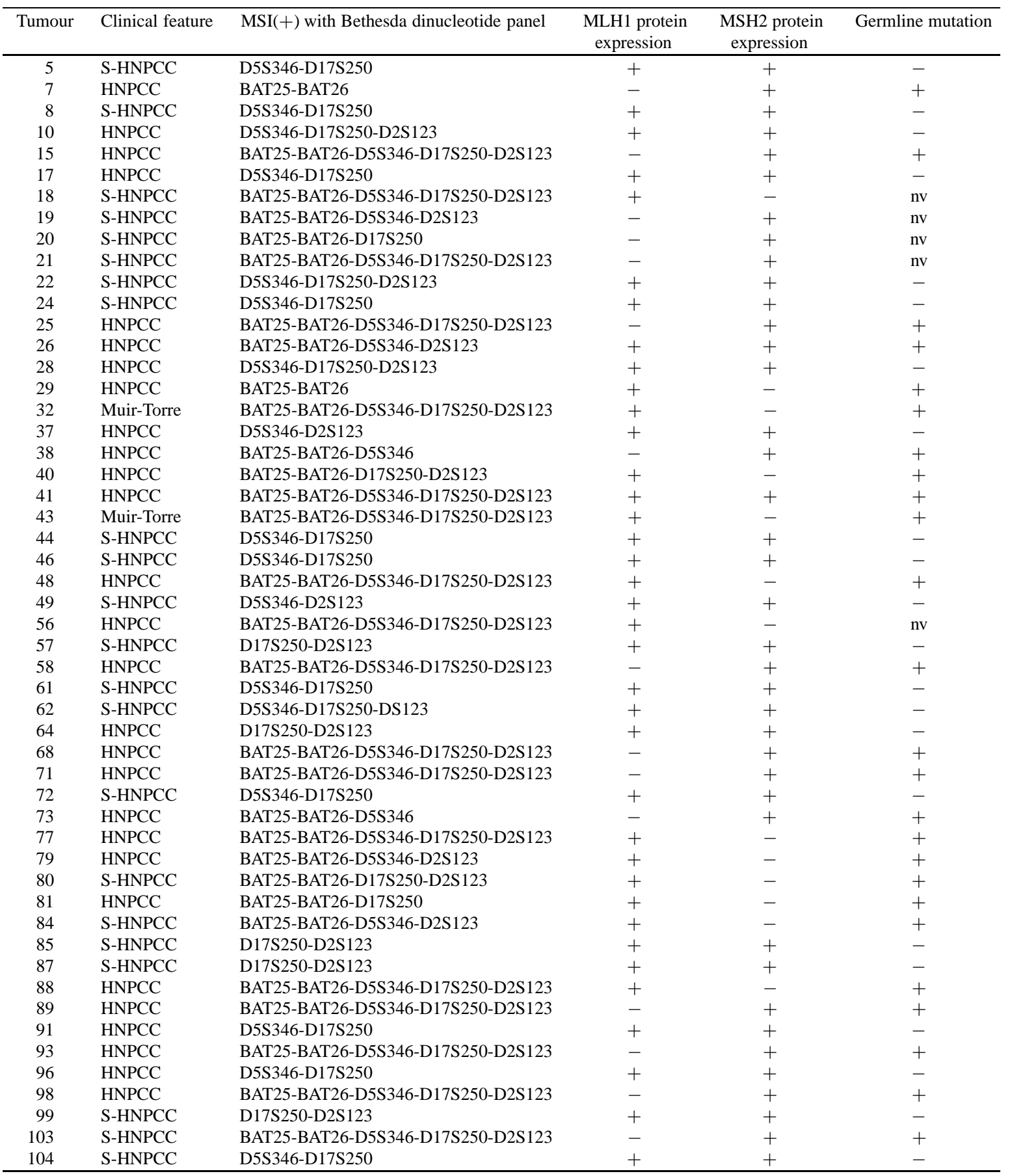

not evaluated $=$ nv, Suspected HNPCC $=$ S-HNPCC.

dard. Specificity was defined as percentage of subjects without mutation who have a negative test result and sensitivity as percentage of subjects with mutation who have a positive test result. The "tests" were MSI anal- ysis with Bethesda or mononucleotide panel, immunohistochemical analysis and single mono/dinucleotide markers. Statistical analysis was carried out using the SPSS 10.0 statistical software (SPSS, Chicago, IL). 
Table 4

Specificity and sensitivity of MSI and IHC in predicting the presence of germline mutations in HNPCC or suspected HNPCC families

\begin{tabular}{lcc}
\hline & Specificity $(\%)$ & Sensitivity $(\%)$ \\
\hline Bethesda markers panel & $70.7(53 / 75)$ & $100(25 / 25)$ \\
Mononucleotide markers panel & $100(75 / 75)$ & $100(25 / 25)$ \\
Immunohistochemical analysis & $100(75 / 75)$ & $92.0(23 / 25)$ \\
BAT25 marker & $100(75 / 75)$ & $100(25 / 25)$ \\
BAT26 marker & $100(75 / 75)$ & $100(25 / 25)$ \\
D2S123 marker & $81.3(61 / 75)$ & $80.0(20 / 25)$ \\
D5S346 marker & $72.0(54 / 75)$ & $80.0(20 / 25)$ \\
D17S250 marker & $64.0(48 / 75)$ & $72.0(18 / 25)$ \\
\hline
\end{tabular}

\section{Results}

The 105 primary colorectal carcinomas were evaluated for MSI status by using both the Bethesda marker panel and the alternative mononucleotide panel. The results are summarized in Table 1 . With the Bethesda panel, 52 tumours $(49.5 \%)$ were found to be positive for two or more markers (high MSI or MSI-H). Using the alternative mononucleotide panel, 30 out of $105(28.6 \%)$ tumours could be classified as MSI $(+)$ $(p<0.001)$. Moreover, with the Bethesda panel 14 tumours (13.4\%) could be scored as MSI-L, whereas no tumour appeared unstable at only 1 marker with the mononucleotide panel. Out of the $52 \mathrm{MSI}(+)$ tumours detected with the Bethesda panel, 28 (54\%) showed lack of expression in one of the two main MMR proteins (MLH1 or MSH2). In contrast, 28 of $30 \mathrm{MSI}(+)$ tumours detected by the alternative mononucleotide markers (93\%) showed lack of expression of MSH2 or MLH1. Thus, mononucleotide markers were significantly more efficient $\left(F_{1}=13.70 p<0.001\right)$ in detecting $\mathrm{MSI}(+)$ tumours associated with lack of expression of MMR proteins. Moreover, protein expression (MLH1/MSH2/MSH6) was normal in the 14 tumours defined as MSI-L (with the Bethesda markers). Thus, the results suggest that the Bethesda panel is probably preferable for detecting all tumours with microsatellite instability (including those with MSI-L), whereas mononucleotide markers instability seems more closely associated with lack of expression of the MMR proteins.

Tables 2 and 3 show the relationship between instability, lack of expression of MSH2/MLH1 proteins and constitutional mutations. With mononucleotide markers, germline mutations were found in all cases with $\mathrm{MSI}(+)$ tumours and lack of expression in one of the MMR proteins (with two exceptions, in which both the main proteins were normally expressed). In contrast, in almost half of the cases assessed with the Bethesda panel, microsatellite instability was not associated with lack of expression of MMR proteins or constitutional mutations. Thus, the Bethesda and the mononucleotide panel showed similar sensitivity in predicting the presence of germline MMR gene defects (25 of 25, 100\%), but a different specificity, which was much higher for the mononucleotide panel (Table 4).

Germline mutations in either $h M L H 1$ or $h M S H 2$ genes could be analyzed in 47 of 52 patients with MSI(+) tumours. Three out of the 5 remaining patients showed no expression of both MLH1 and MSH6 proteins and hypermethylation of the MLH1 promoter region. Constitutional defects were detected in 25 patients from 20 different families; mutations in the $h M S H 2$ gene were found in 11 of them, while in the remaining 9 families MLH1 gene mutations were detected. Clinical features (HNPCC, suspected HNPCC or Muir-Torre syndrome), MSI, immunohistochemistry, molecular changes and their consequence are illustrated in Table 5. One of these molecular changes (ins $\mathrm{T}$ at 2269) was found in the constitutional DNA of 4 apparently unrelated large families living in the area of Modena and Reggio Emilia, Northern Italy. This finding has been interpreted as a "Founder" effect [18]. Rather interestingly, no germline mutations could be detected in patients with MSI-H tumours defined only through dinucleotide instability (i.e., with stable mononucleotide). This last observations further emphasizes the role and significance of mononucleotide markers in detecting HNPCC (or constitutional mutations in the main MMR genes).

\section{Discussion}

The main objective of this study was to develop a simple, rapid and more accurate method of MSI assessment for the identification of HNPCC families. For this reason, we evaluated the efficacy of the Bethesda markers versus a mononucleotide marker panel, composed 


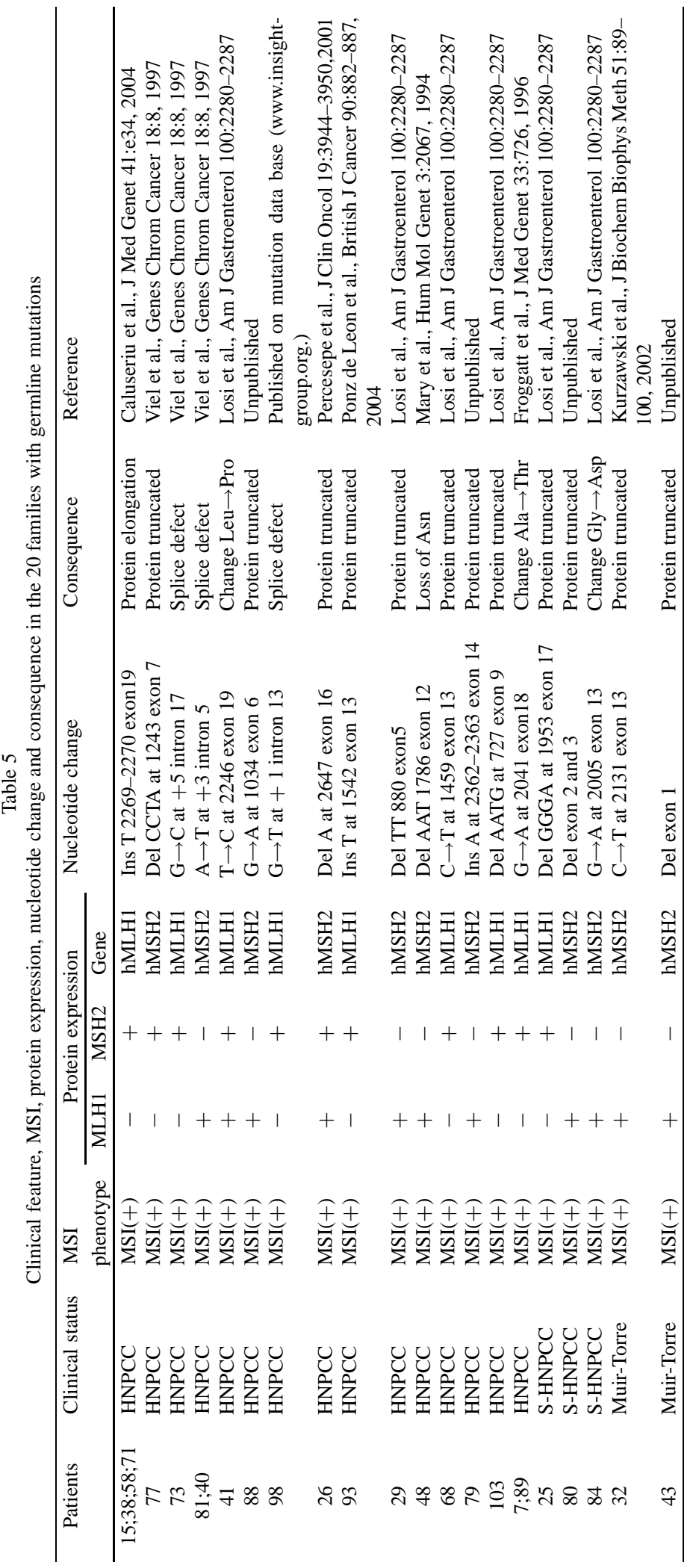


by BAT25 and BAT26, in detecting $h M L H 1 / h M S H 2$ germline mutations.

The results of our study showed that mononucleotide markers are more specific to identify $h M L H 1 / h M S H 2$ mutation carriers than the Bethesda panel (100\% versus $70.7 \%$, respectively). Main limitations of the Bethesda panel are the result of including dinucleotide repeats, since these are less sensitive and less specific for the identification of tumours with no expression of MMR protein and no germline mutation in $h M S H 1 / h M S H 2$ gene, as compared with mononucleotide repeats. Although using the Bethesda markers a tumour with instability at two loci is considered MSI-H, this is not necessarily true if the instability is detected only with dinucleotides. There is evidence [19] suggesting that when enough dinucleotide markers are tested almost all colorectal tumours will show some degree of instability. In our study, we found $22 \mathrm{MSI}-\mathrm{H}$ tumours at dinucleotide loci with normal expression of MLH1/MSH2 proteins and with no germline mutation detectable in the patients. False positive results with the MSI assay, besides an increased time and costs for the IHC test, may lead to over-treatment and unnecessary psychologic stress for the patients. In contrast, the use of mononucleotide markers -such as BAT25 and BAT26allows accurate evaluation of tumours with MMR deficiency without being hindered by the MSI-L category, whose real existence has yet to be proven, and that does not seem to be a homogeneous group [20]. Therefore, it is tempting to delete this category of tumours, which seem to show clinical features in common with MSS tumours and that are different from the MSI-H status of tumours from HNPCC patients. More recently, Vasen et al. suggested that among tumours with MSI$\mathrm{L}$, those with instability at dinucleotide loci showed more often normal expression of MMR protein than MSI-L tumours with instability at a mononucleotide locus [21]. In our study we detected 14 MSI-L tumours where the single unstable repeat was a dinucleotide, and MLH1/MSH2/MSH6 proteins were always normally expressed. Moreover, some MSI tumours with MMR deficiency caused by $h M S H 6$ mutations did not show alterations at dinucleotide repeats [22]. It is important to consider that the MSH6 protein is not involved in the mismatch repair of two or more base pairs, so that the analysis with dinucleotide markers may not recognize many tumours [23]. Thus, we suggest that the use of mononucleotide markers - such as BAT25 and BAT26 could be particularly useful to identify tumours with germline mutations of $h$ MSH6.

In recent years several authors underlined the advantages of IHC over MSI testing [24-26]. IHC was reported to have high sensitivity, to be less expensive and more rapid than MSI testing. Moreover, the loss of one of the MMR proteins indicates which gene is likely to be involved in the disease. Our results demonstrate that IHC with the two commonly used monoclonal antibodies against MLH1 and MSH2 proteins detected a significant fraction of tumours (23 of 25 ) resected from germline mutation carriers. The sensitivity and specificity of IHC in predicting $h M L H 1 / h M S H 2$ germline mutations was $92 \%$ and $100 \%$, respectively, whereas MSI testing showed $100 \%$ in both sensitivity and specificity. Thus, we suggest that IHC can be used as an additional screening procedure in order to identify patients with MMR germline mutations. However, the existence of a small number of cases harbouring a disease-causing mutation, exhibiting $\mathrm{MSI}(+)$ phenotype, and showing normal expression of MMR protein indicates that IHC can hardly replace MSI testing. For this reason, we prefer MSI analysis as the initial step in clinically selected families. In MSI(+) tumours, IHC should be carried out as a second step and, if a negative staining pattern is found, mutation analysis of the involved gene should be the final step.

In conclusion, MSI testing is becoming more and more popular than in the past and it is likely that this assay will be of routine in many laboratories. If we are interested in detecting the MSI status in general, for example because MSI $(+)$ tumours seem to have a more favourable clinical outcome [27], and could be treated differently from MSS tumours, then the Bethesda markers are probably those of choice. However, if a researcher is interested in looking for HNPCC among patients with apparently sporadic colorectal cancer (i.e., the molecular screening frequently advocated by de la Chapelle and collaborators) [28], then the highly more sensitive (in identifying HNPCC) and specific mononucleotide markers - as those proposed in our panel - are probably more suitable for this purpose.

\section{Acknowledgments}

The Authors wish to thank the Italian Association for Cancer Research (AIRC), the Italian Ministry of Education (MIUR), the Consiglio Nazionale delle Ricerche (CNR) and the region Emilia-Romagna for supporting this investigation.

\section{References}

[1] H.T. Lynch and T. Smyrk, Hereditary Nonpolyposis Colorectal Cancer (Lynch syndrome). An updated review, Cancer 78 (1996), 1149-1167. 
[2] J. Jiricny and M. Nystrom-Lahti, Mismatch repair defects in cancer, Curr Opin Genet 10 (2000), 157-161.

[3] R. Fishel, M.K. Lescoe, M.R. Rao et al., The human mutator gene homolog MSH2 and its association with hereditary nonpolyposis colon cancer, Cell 75 (1993), 1027-1038.

[4] M. Miyaki, M. Konishi, K. Tanaka et al., Germline mutation of MSH6 as the cause of Hereditary Nonpolyposis Colorectal Cancer, Nat Genet 17 (1997), 271-272.

[5] P. Peltomaki and H.F.A. Vasen, Mutations predisposing to Hereditary Nonpolyposis Colorectal Cancer: database and results of a collaborative study. The International Collaborative Group on Hereditary Nonpolyposis Colorectal Cancer, Gastroenterology 113 (1997), 1146-1158.

[6] K.W. Kinzler and B. Vogelstein, Lesson from hereditary colorectal cancer, Cell 87 (1996), 159-170.

[7] L.A. Aaltonen, P. Peltomaki, F.S. Leach et al., Clues to the pathogenesis of familial colorectal cancer, Science 20 (1993), $812-816$.

[8] A. Umar, R. Boland, J.P. Terdiman et al., Revised Bethesda Guidelines for Hereditary Nonpolyposis Colorectal Cancer (Lynch syndrome) and Microsatellite Instability, J Natl Cancer Inst 96 (2004), 261-268.

[9] S.B. Hatch, H.M. Lightfood, C.P. Garwacki et al., Microsatellite Instability testing in colorectal carcinomas: choice of markers affects sensitivity of detection of mismatch repairdeficient tumors, Clin Cancer Res 11 (2005), 2180-2187.

[10] C.R. Boland, S.N. Thibodeau, S.R. Hamilton et al., A National Cancer Institute Workshop on Microsatellite Instability for cancer detection and familial predisposition. Development of international criteria for the determination of Microsatellite Instability in colorectal cancer, Cancer Res 58 (1998), 52485257.

[11] M. Perucho, Correpondence re: C.R. Boland et al., A national Cancer Institute Workshop on Microsatellite Instability for cancer detection and familial predisposition: development of international criteria for the determination of Microsatellite Instability in colorectal cancer, Cancer Res 58 (1998), 52485257. Cancer Res 59 (1999), 249-256.

[12] P. Peltomaki, Role of DNA Mismatch Repair defects in the pathogenesis of human cancer, J Clin Oncol 21 (2003), 11741179.

[13] R. Gryfe, H. Kim, E.T.K. Hsieh et al., Tumor Microsatellite Instability and clinical outcome in young patients with colorectal cancer, $N$ Engl J Med 342 (2000), 69-77.

[14] W.S. Samowitz, K. Curtin, K.N. Ma et al., Microsatellite instability in sporadic colon cancer is associated with an improved prognosis at the population level, Cancer Epidemiol Biomarkers Prev 10 (2001), 917-923.

[15] P. Benatti, R. Sassatelli, L. Roncucci et al., Tumour spectrum in Hereditary Non-polyposis Colorectal Cancer (HNPCC) and in families with "suspected HNPCC". A population-based study in northern Italy. Colorectal Cancer Study Group, Int J Cancer 54 (1993), 371-377.

[16] H.F.A. Vasen, P. Watson, J.P. Mecklin et al., New clinical criteria for Hereditary Nonpolyposis Colorectal Cancer (HNPCC, Lynch syndrome) proposed by the International Collaborative Group on HNPCC, Gastroenterology 116 (1999), 1453-1456.

[17] J.G. Park, H.F.A. Vasen, Y.J. Park et al., Suspected HNPCC and Amsterdam criteria II: evaluation of mutation rate, an international collaborative study, Int J Colorectal Dis 17 (2002), 109-114.

[18] O. Caluseriu, C. Di Gregorio, E. Lucci-Cordisco et al., A founder MLH1 mutation in families from the districts of Modena and Reggio Emilia in Northern Italy with Hereditary NonPolyposis Colorectal Cancer associated with protein elongation and instability, J Med Genet 41 (2004), e34.

[19] P. Laiho, V. Launonen, P. Lahermo et al., Low-Level Microsatellite Instability in most colorectal carcinomas, Cancer Res 62 (2002), 1166-1170.

[20] I. Tomlinson, S. Halford, L. Aaltonen et al., Does MSI-Low exist? J Pathol 197 (2002), 6-13.

[21] H.F.A. Vasen, Y. Hendriks, A.E. de Jong and et al., Identification of HNPCC by molecular analysis of colorectal and endometrial tumors, Dis Markers 20 (2004), 207-213.

[22] Y. Akiyama, H. Sato, T. Yamada et al., Germ-line mutation of the hMSH6/GTBP gene in an atypical Hereditary Nonpolyposis Colorectal Cancer kindred, Cancer Res 57 (1997), 3920-3923.

[23] N. Papadopoulos, N.C. Nicolaides, B. Liu et al., Mutations of GTBP in genetically unstable cells, Science 268 (1995), 1915-1917.

[24] R. Ward, A. Meagher, I. Tomlinson et al., Microsatellite Instability and the clinicopathological features of sporadic colorectal cancer, Gut 48 (2001), 821-829.

[25] N.L. Lindor, L.J. Burgart, O. Leontovich et al., Immunohistochemistry versus Microsatellite Instability testing in phenotype colorectal tumors, J Clin Oncol 20 (2002), 1043-1048.

[26] A.M. Chiaravalli, M. Cornaggia, D. Furlan et al., The role of histological investigation in prognostic evaluation of advanced gastric cancer. Analysis of histological structure and molecular changes compared with invasive pattern and stage, Virchows Arch 439 (2001), 158-169.

[27] P. Benatti, R. Gafâ, D. Barana et al., Microsatellite Instability and colorectal cancer prognosis, Clin Cancer Res 11 (2005), 8332-8340.

[28] H. Hampel, W.L. Frankel, E. Martin et al., Screening for the Lynch sindrome (Hereditary Nonpolyposis Colorectal Cancer), N Engl J Med 352 (2005), 1851-1860. 


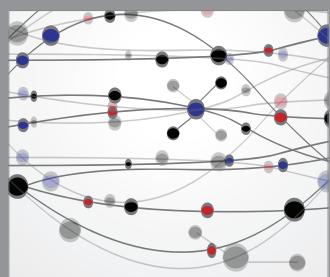

The Scientific World Journal
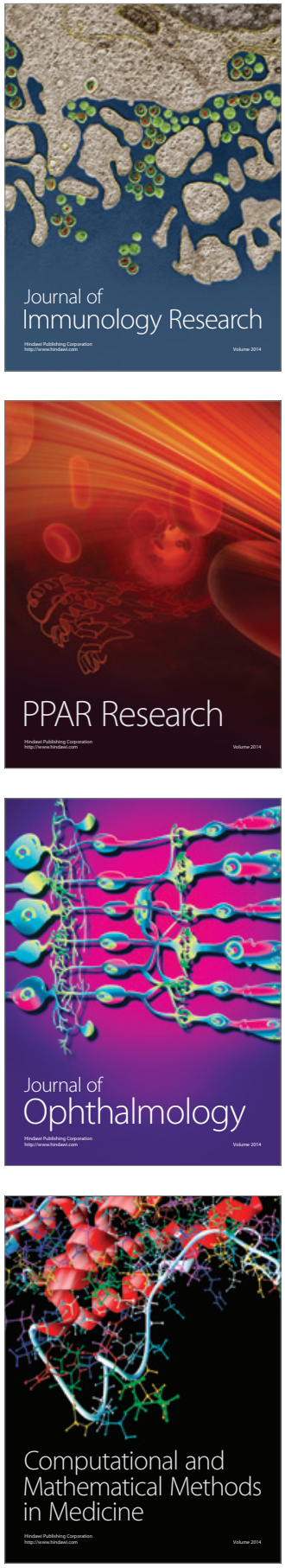



Gastroenterology

Research and Practice
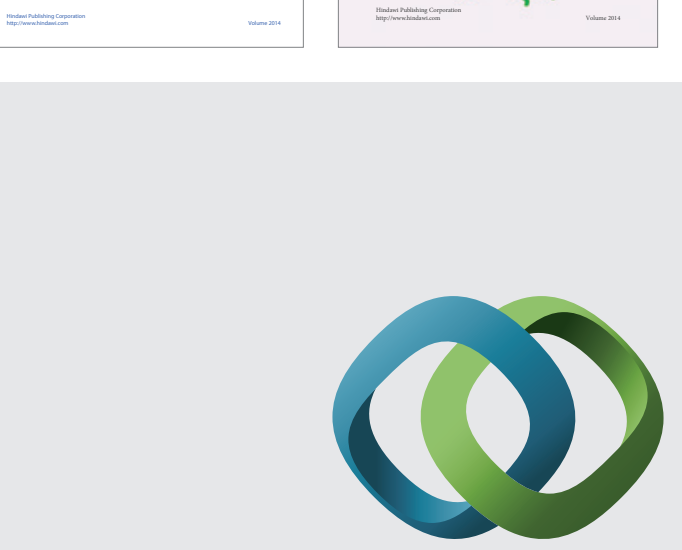

\section{Hindawi}

Submit your manuscripts at

http://www.hindawi.com
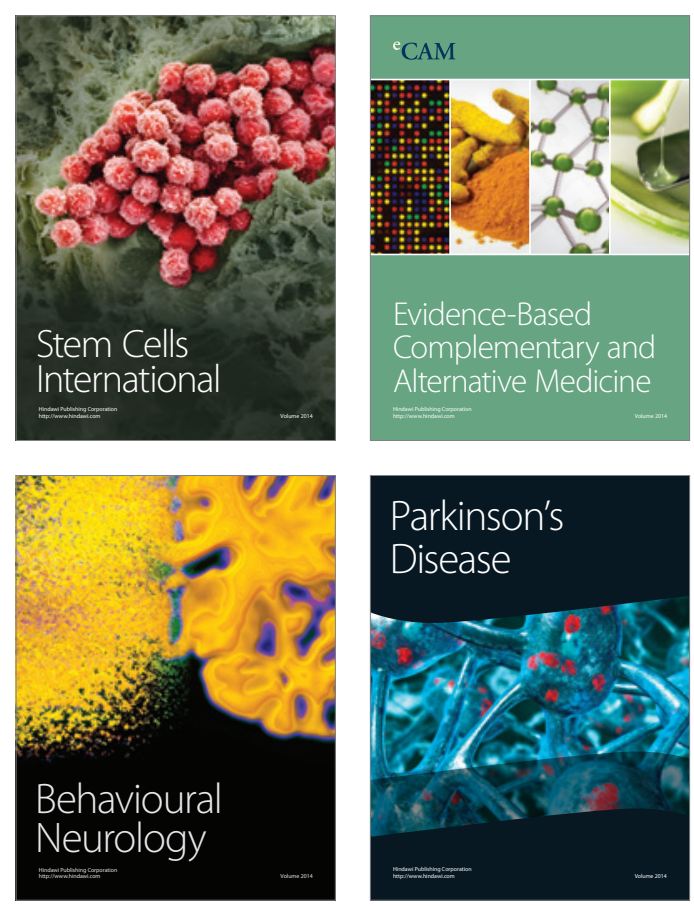



Journal of
Diabetes Research



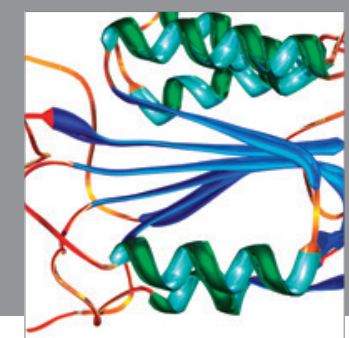

Disease Markers
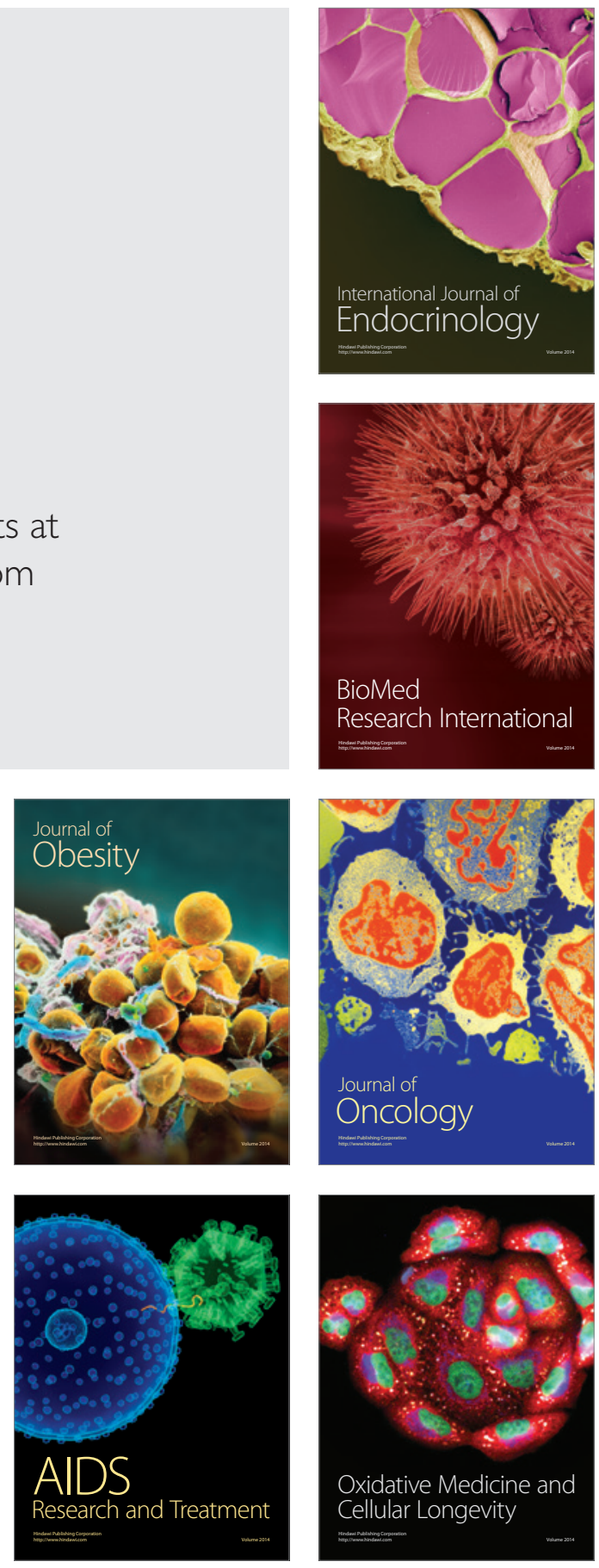